\title{
MOEAs in the Design of Network Centric Systems (Abstract of Invited Talk)
}

\author{
Gary B. Lamont \\ Air Force Institute of Technology \\ 2950 Hobson Way, Wright-Patterson AFB, Dayton, OH 45433-7765, USA \\ Gary. Lamont@afit.edu
}

\begin{abstract}
Advances in information and communications technology are changing network design techniques quantitatively and qualitatively. This technology is supporting the design of large scale network centric systems which are required in many contemporary real-world situations. These highlevel robust centric systems by definition must provide improved information sharing and collaboration between network entities. Such systems enhance the quality of information awareness, improving sustainability, and mission effectiveness and efficiency. The hierarchical development of network centric systems includes all dynamic information elements and is applied so as to maximize the desired decision and action impact. Associated network information flow problems can have as objectives costs, delays, robustness, vulnerability, and reliability with related constraints of network flow capacities, rates, and quantities of information. The optimization of coupled complex capacitated network flow problems is therefore an integral and basic element of network centric systems design. Thus, the focus of the discussion is on the efficacy of multiobjective evolutionary algorithms (MOEAs) to solve effectively and efficiency variations of associated network flow problems, given sophisticated mathematical models. Also to be addressed are dynamic network environments where various information channels become nonavailable, change their characteristics, or information priorities are modified. Discrimination between possible MOEA operators (recombination, mutation, selection) and associated MOEA parameter values is discussed as related to solving effectively variations of multiobjective network centric information flow problems including real-time behavior. Example network flow applications provide insight to choosing appropriate MOEA characteristics. Included is a discussion of opportunities for future MOEA research in this arena.
\end{abstract}

